\title{
PARTIDA DA TURBINA DE TOPO DO AF3*
}

\author{
André Luis de Oliveira ${ }^{7}$ \\ Alcides Jose de Lucena Silva ${ }^{2}$ \\ Lisandro Pegorim Miller ${ }^{3}$
}

\section{Resumo}

O Alto Forno 3 (AF3) da Companhia Siderúrgica Nacional (CSN) é o equipamento muito importante no processo siderúrgico da Usina Presidente Vargas (UPV), situada em Volta Redonda (RJ), por responder por mais de $70 \%$ da produção de gusa da UPV. Na produção de gusa é gerado o Gás de Alto Forno (GAF), subproduto de alta relevância, pois este gás sai do topo do AF3 com grande pressão e cinética, energia essa passível de utilização. Em 2007 a CSN, optou por adquirir um equipamento denominado "Top Pressure Recovery Turbine" (TRT), capaz de aproveitar tal energia para gerar energia elétrica. Em 2013, a equipe de supervisores da $\mathrm{KHI}$ - Kawasaki Heavy Industrie, fabricante da turbina, veio para o Brasil para realizar no equipamento os testes a frio e a quente para na seqüência vir a operá-lo. Ocorre que fatores alheios ao objetivo desta apresentação impediram a equipe da $\mathrm{KHI}$ de partir o equipamento no tempo previsto, bem como de retornar ao Brasil para dar seqüência nas atividades. Em novembro de 2013 a CSN definiu por partir o equipamento com recursos próprios, sendo este o foco do trabalho. A presente contribuição técnica vem mostrar o trabalho desenvolvido internamente pelo grupo técnico a fim de finalizar o projeto e colocar a Turbina de Topo (TRT) em operação, fato este dado total ênfase pela direção da empresa devido ao cenário nacional de crise energética em que os valores de energia estavam no limite máximo permitido pela legislação e impactando diretamente nos custos da empresa.

Palavras-chave: Alto forno 3; Turbina de topo; Partida.

\section{START OF TURBINE TOP OF BLAST FURNACE 3}

\section{Abstract}

The Blast Furnace 3 of the Companhia Siderúrgica Nacional (CSN) is the very important equipment in the steelmaking process, located in Volta Redonda (RJ), for accounting for over $70 \%$ of the pig iron production of CSN. In the production of pig iron is generated Blast Furnace Gas (BFG), a byproduct of high relevance, as the gas exits the top of the AF3 with great pressure and cinetic energy liable to use this. In 2007, CSN, chose to purchase a device called "Top Pressure Recovery Turbine" (TRT), capable of harnessing such energy to generate electricity. In 2013, the team of supervisors KHI - Kawasaki Heavy Industrie, the turbine manufacturer, came to Brazil to perform equipment testing cold and hot to the sequence come to operate it. Occurs that factors beyond the scope of this presentation prevented the team from $\mathrm{KHI}$ of the equipment on time as well as returning to Brazil to follow up on activities. In November 2013 the CSN defined by the equipment from its own resources, which is the focus of the work. This technical contribution is to show the work done internally by the technical group in order to complete the project and put the top turbine (TRT) in operation, a fact emphasized by the overall direction of the company due to the national energy crisis scenario in which energy values were the maximum extent permitted by law and directly impacting the company's costs.

Keywords: Blast furnace; Top pressure recovery; Start.

Engenheiro Especialista, Gerência de Geração de Energia, Companhia Siderúrgica Nacional (CSN), Volta Redonda, Rio Janeiro, Brasil.

Engenheiro Desenvolvimento, Gerência Alto Forno, CSN, Volta Redonda, Rio de Janeiro, Brasil.

Engenheiro Especialista, Gerência Geral Engenharia, CSN, Volta Redonda, Rio de Janeiro, Brasil. 


\section{INTRODUÇÃO}

O Alto Forno 3 da CSN responsável pela produção de $70 \%$ do gusa da UPV é foco na busca do melhor aproveitamento dos resíduos gerados (Gás de Alto Forno, escória, pó e lama, etc.) visando sempre a melhor eficiência do processo bem como o aproveitamento de energias latentes no sistema.

No processo do Alto Forno, a energia provida do Ar soprado (oxigênio) é utilizada para prover o aquecimento do combustível (coque) a fim de fundir a matéria prima para produção do gusa. Este Ar Soprado sai do alto forno em forma de Gás Siderúrgico (Gás de Alto Forno), sendo utilizado em toda a UPV nos processos siderúrgicos no aquecimento dos mesmos.

O estudo oriundo do ano 2007 visou o aproveitamento da energia cinética contida na saída do Alto Forno devido a grande vazão do mesmo $\left(+/-6500 \mathrm{Nm}^{3}\right)$ a uma pressão de (+/- 2,6kg). O projeto da instalação de uma Turbina de Topo daria um destino rentável para esta energia que estava sendo desperdiçada.

Nessa linha, em 2007, a CSN abriu um processo para adquirir um equipamento, denominado TRT - Top Pressure Recovery Turbine, capaz de realizar tal transformação. Assim, 09/10/07, a CSN contratou a KHI - Kawasaki Heavy Industries, que ofertou uma TRT com capacidade de gerar até $21 \mathrm{MWh}$, se consideradas as condições operacionais do AF3 previstas em projeto.

No período de 27/02/13 a 22/03/13, a equipe de supervisores da KHI, juntamente com os técnicos da GGEN, veio ao Brasil para realizar os testes a frio e a quente após todas as etapas de montagem civil, eletromecânica e auxiliar em geral para comissionamentos a Frio e a Quente - além de colocar a TRT em operação.

Mas, atrasos no Comissionamento a Frio, não computáveis à CSN, impediram os objetivos acima e, além disso, por fatores alheios ao objetivo deste trabalho, a equipe KHI não pôde retornar ao Brasil.

Tal situação levou que em final de novembro/13, a diretoria CSN solicitou uma análise afim de que a TRT fosse posta em marcha (operação), sem a supervisão da $\mathrm{KHI}$.

Entre as razões que levaram a esta tomada de decisão podemos citar:

a) TRT 100\% montada;

b) Atratividade de mercado para a geração própria de energia elétrica ainda maior que à época da concepção do projeto;

c) Confiança em seus técnicos para vencer o desafio.

Assim, iremos verificar no desenvolvimento do trabalho as atividades que levaram a Turbina de Topo do AF3 a estar em operação desde 15/01/2014.

\section{OBJETIVOS}

Disponibilizar a Turbina de Topo em condições operacionais devido ao grande impacto econômico da mesma nos negócios da CSN mantendo a integridade da máquina e garantindo a segurança das pessoas.

\section{METODOLOGIA}

A definição das estratégias para condução dos trabalhos com as GG's envolvidas passava por determinar quais as especialidades e focos de cada uma, para definir e levantar todos os questionamentos necessários bem como os recursos físicos e técnicos para o trabalho. 
As etapas das quais se baseou o desenvolvimento foi o estudo de toda a operacionalidade da Turbina bem como as interfaces existentes para levantar gargalos e nortear a necessidade de ajuda externa (Brasil) de usinas que já possuíam a Turbina Implantada e/ou com o Know-how da própria equipe técnica interna.

\section{DESENVOLVIMENTO}

\subsection{Descrição Funcional da TRT}

A TRT (Turbina de Topo de Alto Forno) é um equipamento projetado para gerar energia elétrica através do gás de Alto Forno (GAF) do topo de um Alto Forno, aproveitando de características termodinâmicas como: temperatura, pressão e volume.

A expansão controlada do gás produzido internamente no Alto Forno nํㅜ da CSN é feita através da TRT produzindo energia elétrica, cuja quantidade é em função de características do gás na entrada da turbina, tendo uma vazão média de 540.000 $\mathrm{Nm} 3 / \mathrm{h}$ com uma pressão de controle de topo média de $2,20 \mathrm{~kg} / \mathrm{cm}^{2}$ à uma temperatura na faixa de 45 a $55^{\circ} \mathrm{C}$.

As características do gás na saída da turbina apresentam pressão de controle de $0,10 \mathrm{~kg} / \mathrm{cm} 2$ e uma temperatura na faixa de $60^{\circ}$. O rendimento da turbina é em função das variações de vazão de BFG que podem ocorrer diariamente na operação do Alto Forno.

Anteriormente esta energia era desperdiçada na necessária redução da pressão do gás de Alto Forno (GAF) para armazenamento e distribuição, além de gerar ruído e aquecimento na válvula responsável pela função de controlar a pressão no topo do Alto Forno.

Este processo de recuperação de energia interrompe o desperdício continuado existente há mais de 15 anos de operação do Alto Forno na CSN.

\subsection{Construção Civil, Montagem Eletromecânica e Automação.}

Por se tratar de equipamento de grande porte e ainda, que opera em altíssima velocidade de rotação (3600 rpm), a construção das bases e posicionamento dos chumbadores, obedeceram rigorosamente às dimensões e posicionamentos do projeto executivo.

Por ser um equipamento complexo e com sofisticada estrutura de segurança e de proteção, o conjunto Turbina/Gerador está monitorado por 3 (três) estações de controle lógico redundantes (PLCs), que recebem sinais diretamente das partes móveis e estratégicas. Estes equipamentos operam com restritas faixas de tolerâncias (limites de temperaturas, vibrações, velocidades, tensões e correntes elétricas, dentre outras).

Os sensores e ligações "via fibra ótica", são considerados os principais elementos de segurança operacional da Turbina de Topo e, qualquer anormalidade que afete os limites preconizados em projeto, leva imediatamente ao desligamento automático e isolamento dos equipamentos da Turbina de Topo. 

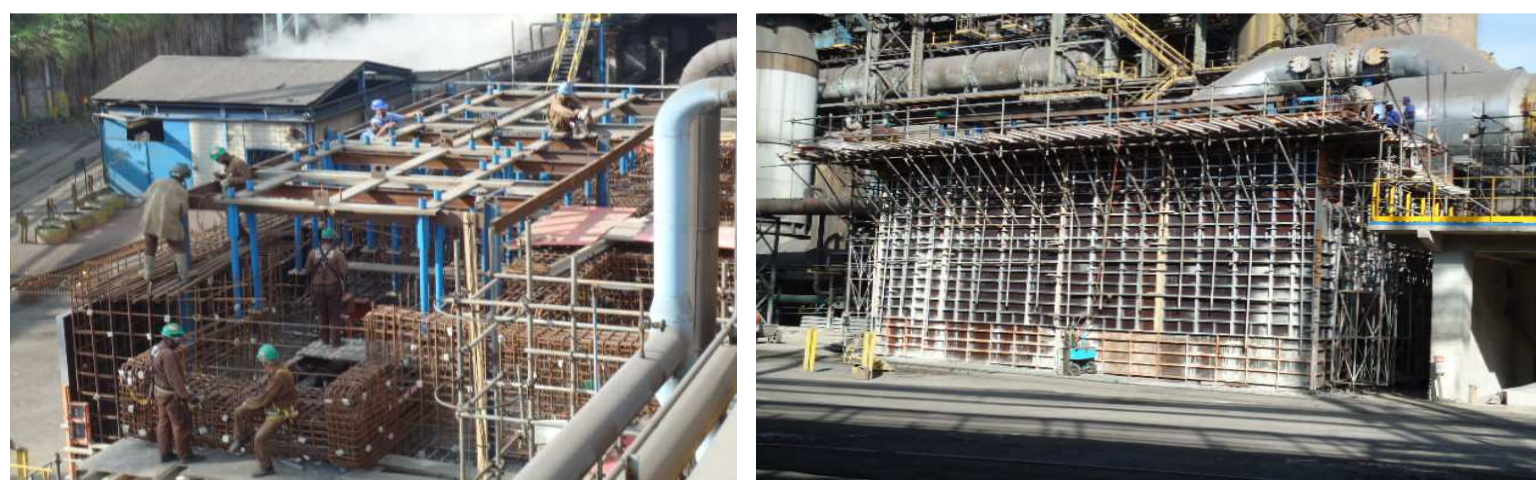

Figuras 1 e 2. Ferragem, chumbadores e formas da base da Turbina e Gerador

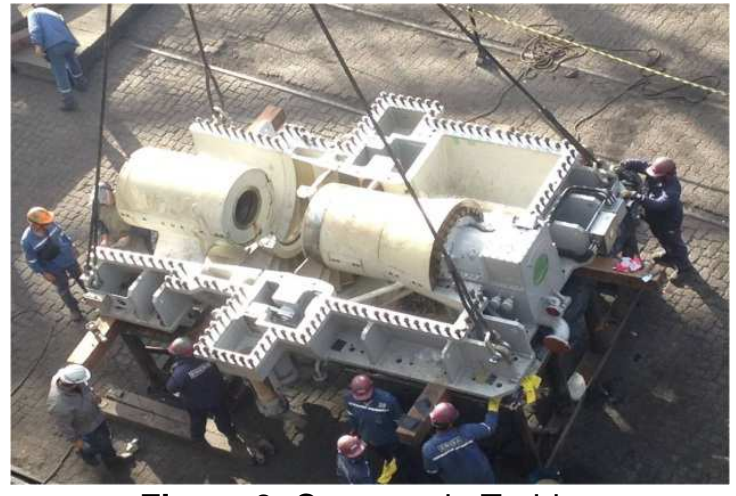

Figura 3. Carcaça da Turbina

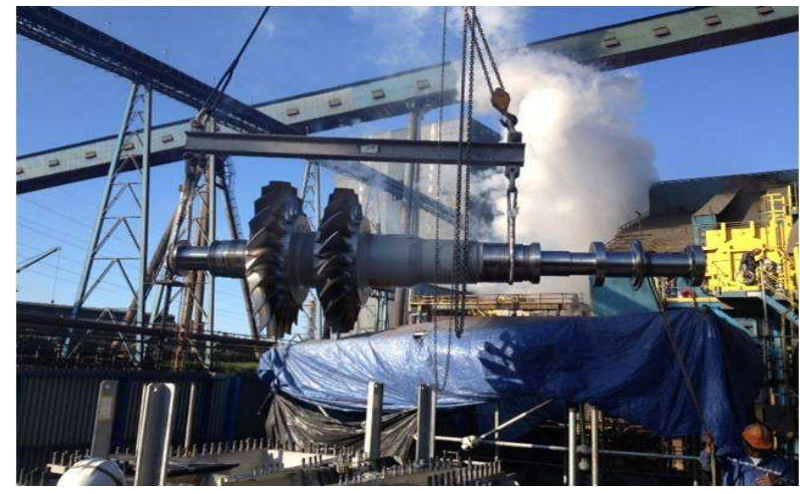

Figura 4. Rotor da Turbina

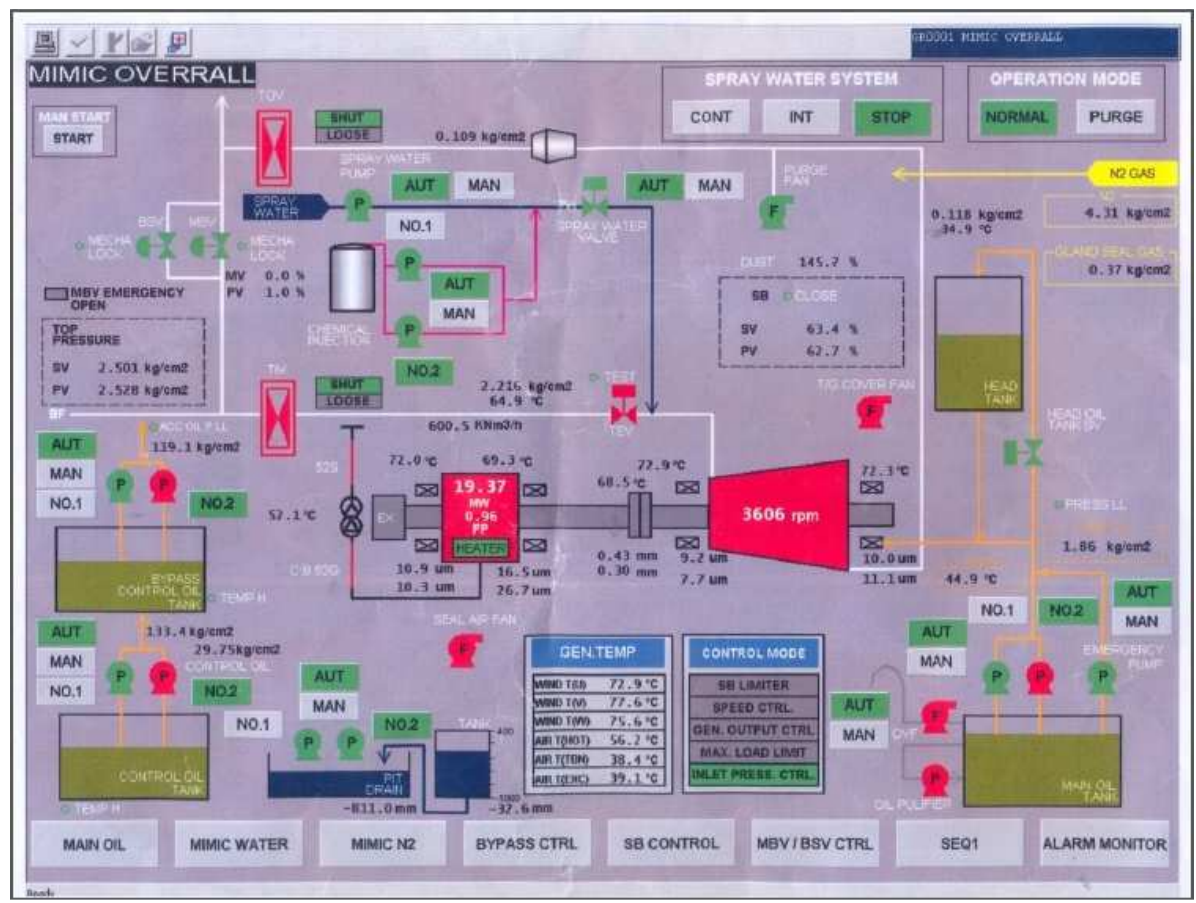

Figura 5. Tela de monitoramento e controle operacional

\subsection{Diagrama Macro da Turbina de Topo e Curva Operacional}

As características técnicas e a curva operacional podem ser vistas na figura 6 abaixo. De acordo com o projeto temos uma vazão máxima de entrada de 
$750.000 \mathrm{Nm}^{3} / \mathrm{h}$ com uma pressão de topo de $2,5 \mathrm{~kg}$ podemos atingir $21 \mathrm{MW}$ de geração de energia elétrica.

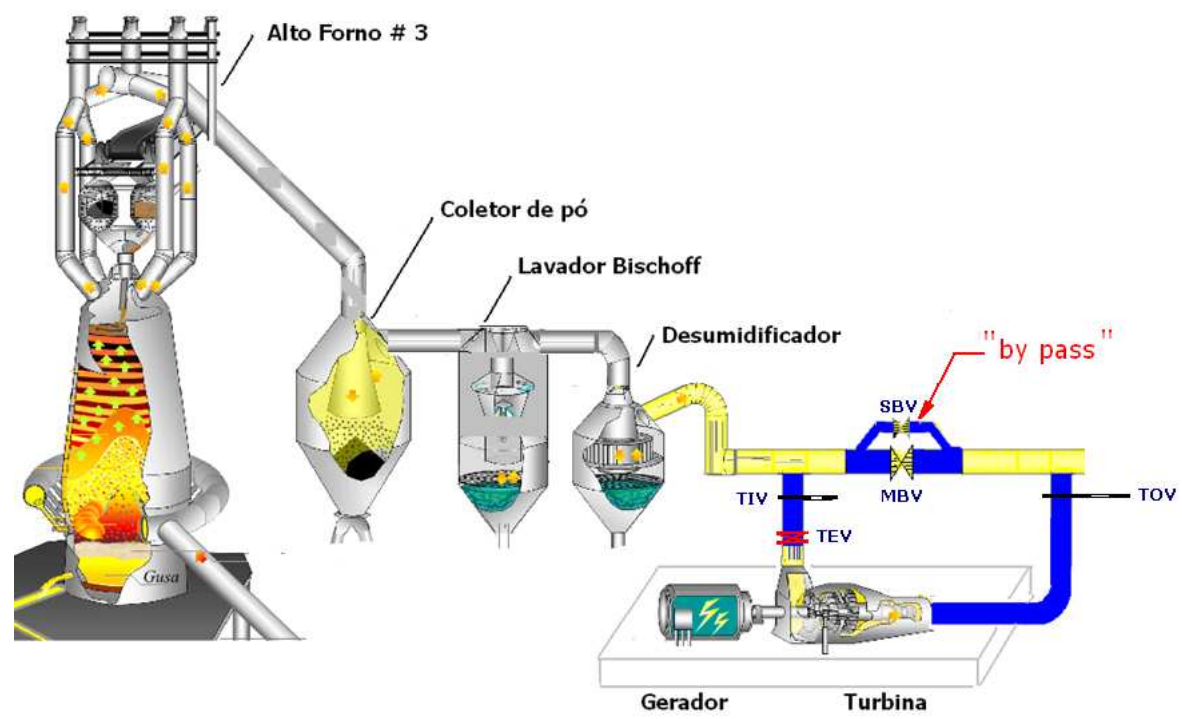

Figura 6. Diagrama funional da TRT x Alto Forno 3 [Fonte: Kawasaki].

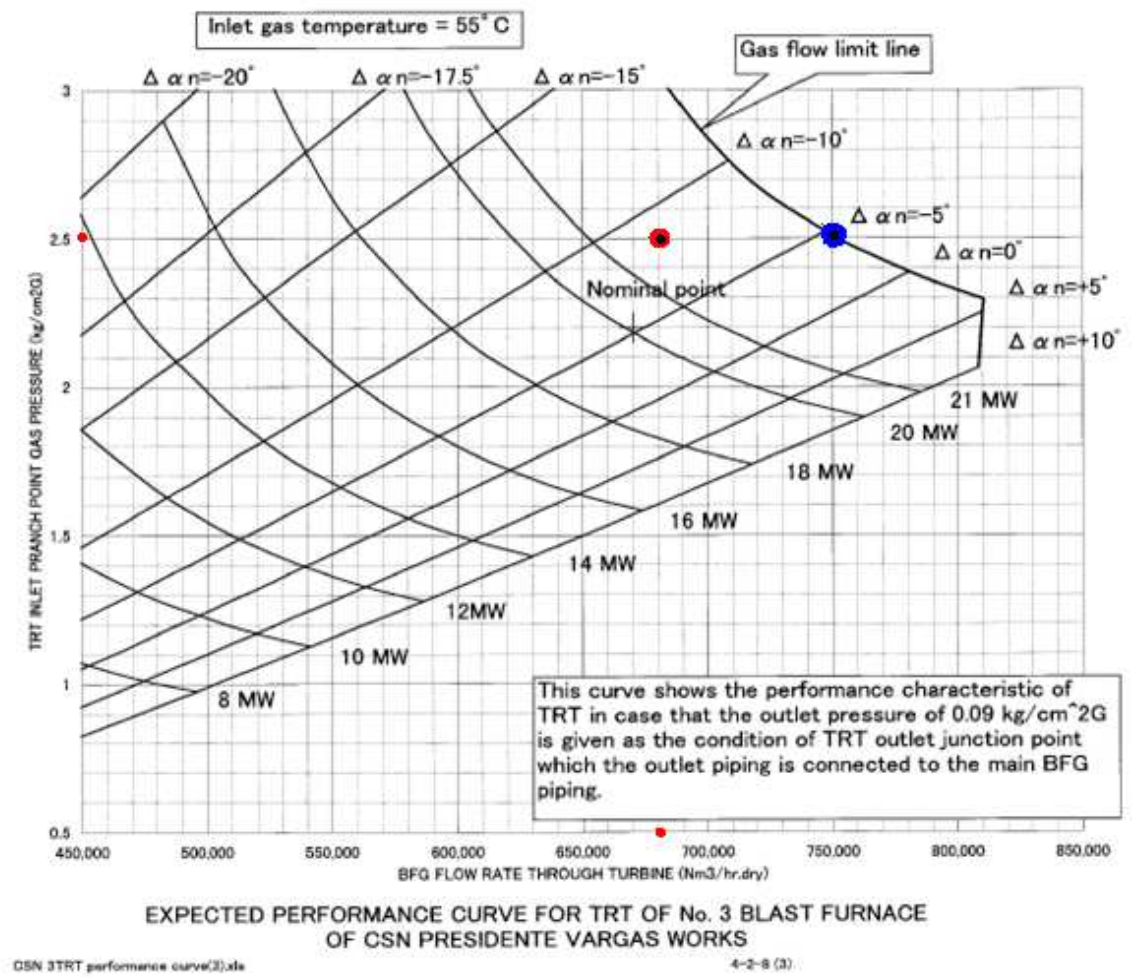

Figura 7. Curvas de performance da TRT Alto Forno 3 CSN [Fonte: Kawasaki]..

\section{DETALHAMENTO DO PROBLEMA}

Em outubro 2007 a CSN contratou a KHI - Kawasaki Heavy Industries, que ofertou uma TRT com capacidade de gerar até $21 \mathrm{MWH}$, se consideradas as condições operacionais do AF3 previstas em projeto. Em 2012, através da empresa contratada pela CSN, foi feita a montagem eletromecânica, sendo concluída no ano 2012.

No período de 27/02/13 a 22/03/13, as equipes de supervisores da $\mathrm{KHI}$, juntamente com os técnicos da GGEN, vieram ao Brasil para realizar os testes a frio e a quente - Comissionamentos a Frio e a Quente - além de colocar a TRT em operação. 
Durante a fase em que os técnicos da Kawasaki estavam na planta, problemas não computáveis a CSN e alheios ao objetivo do trabalho vieram a causar atrasos no Comissionamento a Frio e à quente, que impediram a conclusão do projeto.

Do final de março/2013 até novembro/2013 várias tentativas de retornar com o corpo técnico Kawasaki não obtiveram êxito.

O equipamento TRT era um projeto estratégico pelo nível de investimento e retorno rápido conforme figura 8 .

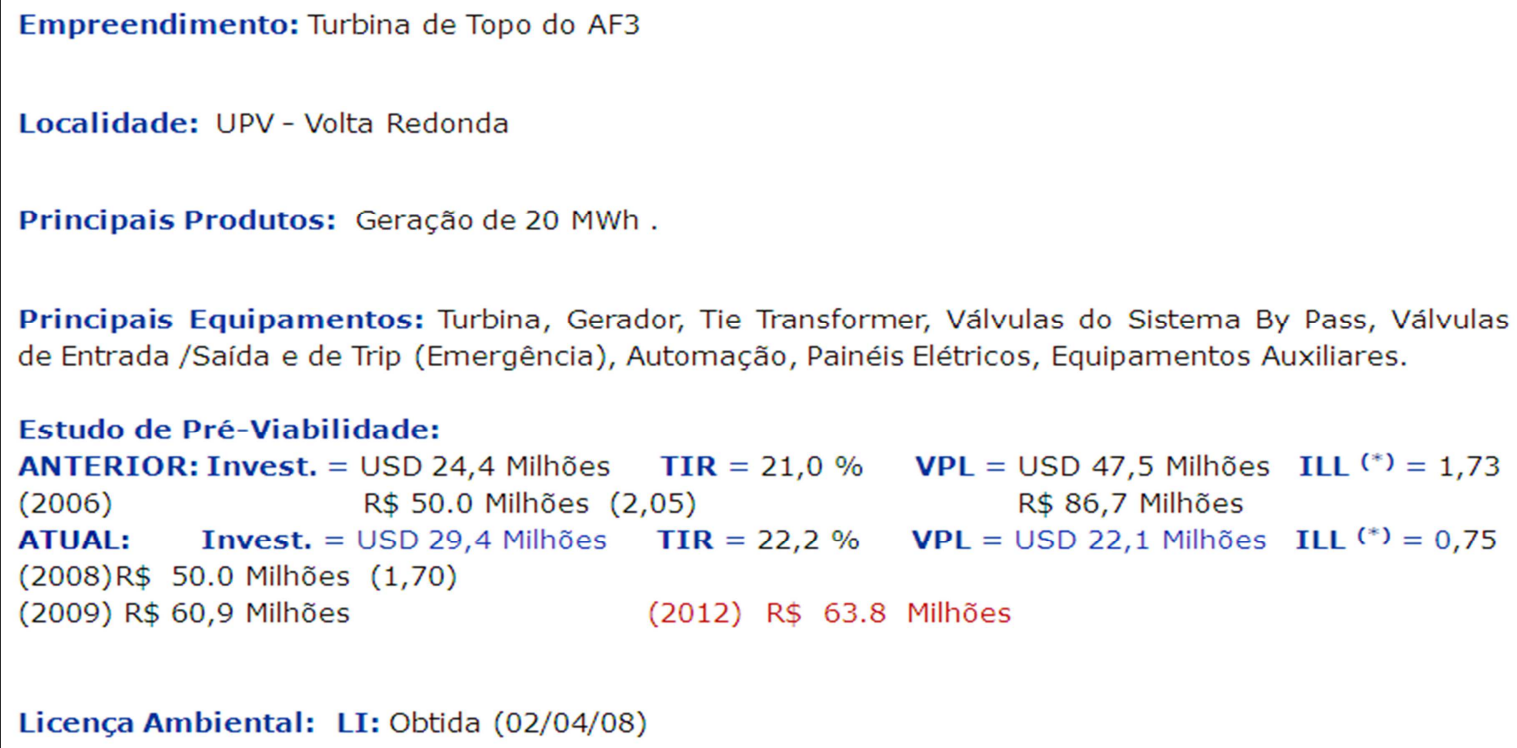

Figura 8. Estudo de viabilidade [Fonte: CSN-GGEN].

A situação em que o mercado de energia elétrica se encontrava (alto custo da energia elétrica) levou a uma decisão em final de novembro/2013. Foi solicitada pela direção da empresa (DEPRO) que se buscasse todos os recursos internos necessários para colocar a TRT em marcha, ou seja, operando sem a supervisão do fabricante (KHI). Podemos citar algumas razões dos quais o DEPRO se baseou:

a) TRT $100 \%$ montada;

b) Atratividade de mercado para a geração própria de energia elétrica ainda maior que à época da concepção do projeto (preço da energia altíssima $>\mathrm{R} \$ 800,00 / \mathrm{MWh})$;

c) Confiança em seus técnicos para vencer o desafio.

Pelos valores de aquisição da energia externa, a falta da TRT levava a uma condição em que se perdia uma grande parcela do retorno do investimento pelo fato dela estar parada. Podemos ter uma ideia bem simplificada deste prejuízo efetuando a seguinte multiplicação:

- Valor da energia de mercado CSN (R\$120,00/ MWh)

- Média esperada de geração 16MW/h

Temos então:

$-120,00 \times(16 \times 24 \mathrm{~h} \times 30$ dias $)=R \$ 1.555 .200,00$ mensais .

Este cálculo será refinado e mostrado o valor real levando em consideração todos os custos diretos e indiretos, mas já é possível ver a magnitude de ganho que estava sendo perdido pela CSN.

Sob a coordenação da GGEN-AF, de posse da solicitação da diretoria, uma equipe técnica multidisciplinar e multigerencial composta pelas gerências envolvidas no 
projeto (GGAF, GGCE, GGEN e GGOS) para analisar e buscar a solução do problema.

O grande desafio da equipe era de assimilar todo conhecimento necessário para executar todos os testes faltantes seguindo o planejamento da Kawasaki e também efetuar a partida da TRT sem a garantia do fabricante, levando a aumentar substancialmente as responsabilidades e o impacto caso ocorresse algum problema no equipamento.

\section{SOLUÇÃO PROPOSTA}

A Equipe partiu com duas linhas de trabalho paralelas a fim de viabilizar a colocação da TRT em operação, sendo elas:

1. Buscar no mercado interno uma assistência técnica de empresas que já possuem o equipamento instalado e em operação;

2. Formar equipes disciplinares (mecânica, elétrica, instrumentação e automação) para estudar e analisar profundamente todas as partes do equipamento e verificar a existência de equipamentos denominados "caixa preta", ou seja, só é possível acessar pelo fabricante.

\subsection{Primeira Linha de Trabalho (Busca de Especialistas em TRT no Mercado Interno)}

A busca por especialistas de outras empresas no equipamento bem como a assistência técnica levou o grupo a efetuar contatos com a USIMINAS, CST, pois possuíam Turbinas de Topo de mesmo fabricante e similares a da CSN. No entanto não havia disponibilidade dos especialistas necessários vir a CSN efetuar o comissionamento e startup da TRT.

\subsection{Segunda Linha de Trabalho (Formação de Equipes Internas por Disciplina)}

A formação das equipes levou cada especialista a detalhar e estudar os processos, suas peculiaridades e também entender profundamente o equipamento e suas interfaces.

Podemos citar pontos especiais da Turbina de Topo:

1. Válvulas controladoras (MBV, BSV)

2. Palhetas da Turbina

3. CLP (Yokogawa)

4. Controlador da Turbina ( $\mathrm{S} 7400 \mathrm{H}$ - Siemens)

5. Proteção de Vibração (Bently Nevada - 3500)

6. Proteção de Sobrevelocidade (Protech - Woodward)

7. Sistema hidráulico de controle

8. Sistema de sincronismo com a rede (Yokogawa)

9. Relés de proteção (Areva)

Cada item acima foi analisado, testado, garantindo a funcionalidade individual de cada equipamento de acordo com o projeto.

Durante cada teste, os questionamentos eram equacionadas através de reuniões técnicas específicas para buscar a solução e a tomada de decisão correta.

A participação da gerência de altos fornos (GGAF) proporcionava a necessidade constante de garantir a integridade e produção do Alto forno 3 mesmo com os testes 
necessários na Turbina de Topo, mitigando os riscos existentes e traçando as contingências.

As atividades previstas no roteiro de comissionamento a frio e à quente foram executadas no período de 02/12/2013 à 06/01/2014 sendo necessário alguns envolvimentos de equipes contratadas.

\section{COMISSIONAMENTOS A FRIO E QUENTE - TESTES PREVISTOS E REALIZADOS}

Inicialmente se pensou na elaboração de um Plano de Testes contemplando apenas aqueles parcialmente concluídos durante o Comissionamento a Frio e todos os do Comissionamento a Quente, exceto a atividade $\mathrm{M}-1$, concluída pela $\mathrm{KHI}$. Mas, o grupo CSN entendeu ser importante a repetição de alguns testes do Comissionamento a Frio e realizar o Comissionamento a Quente a partir da atividade $\mathrm{M}-1$.

\subsection{Comissionamento a Frio}

\section{Testes Mecânicos M-1 a M-9, Instrumentação l-1 e Elétricos E-1 a E-7}

\subsubsection{M-1 Bypass Valve System}

Realizado pela KHI entre 10/12/12 a 21/12/12. Os demais testes abaixo indicados a KHI os realizou em 2 etapas, com a conclusão ocorrendo entre 07/03/13 e 19/03/13. A CSN refez os testes necessários do M-1-1 ao M-1-6 entre 05/12/13 e 13/12/13.

\section{M-1-3 - Ocorrência:}

Ação: Aprox. em Junho/13 - Testes do circuito de água em baixa pressão da refrigeração do AF3, oriunda da CBR4, que também dá origem ao sistema de refrigeração da TRT que indicava queda na pressão da água no sistema.

Problema: Grande divergência entre as pressões real e de projeto.

- Solução: Out/13, na MP AF3 foram trocados os Filtros de Baixa Pressão da CBR\#4, pois já tinha sido identificado que continham uma grande quantidade de detritos nas cestas de filtragem de água. Resolvido.

- Ação: 05/12/13 - Reteste da unidade hidráulica do óleo de controle do SB Stator Blade e válvulas MBV - Main Bypass Valve e SBV - Secondary Bypass Valve.

- Problema:Presença de água no tanque de drenagem de emergência da unidade.

- Solução: 05/12/13 - Drenagem do tanque de emergência.

- Problema:Afluência de água no tanque de emergência acima da sua capacidade de drenagem.

- Solução: 06/12/13 - Adaptação de dispositivo de drenagem com maior capacidade de vazão.

- Problema:Superaquecimento do óleo $\rightarrow$ Temperatura $>47^{\circ} \mathrm{C}$.

- Solução: 13/12/13 - Limpeza das tubos do cooler do sistema de refrigeração do AF3 $\rightarrow$ Regularização da vazão do circuito de baixa pressão da água de refrigeração do cooler da TRT.

- Problema:Uma das bombas de fuso da Unidade Hidráulica de Óleo de Controle apresentou defeito durante testes iniciados em 27/02/13. 
- Solução: Aprox. em Maio/14 - Substituição da bomba danificada e teste do sistema $\rightarrow$ Resolvido.

\subsubsection{M-2 Turbine Inlet and Outlet Shut-off Valves}

Realizado pela KHI entre 13 e 15/12/12 e refeito pela CSN em 09/12/12 e depois em $16 / 12 / 13$

\subsubsection{M-3 Oil and Sealing Gas System for Turbine}

Realizado pela $\mathrm{KHI}$ em 27 e 28/12/12 e refeito parcialmente pela CSN entre 05/12/13 e 13/12/13, por não ser necessário em sua totalidade, devido ao atendimento do programa de preservação determinado pela $\mathrm{KHI}$.

\subsubsection{M-4 Interlock System}

Presente no software da TRT, checado pela KHI em 28/12/12, checado sua integridade pela CSN entre 05/12/13 e 06/12/13 e realizado o teste entre 13/12/13 e $17 / 12 / 13$

\subsubsection{M-5 Emergency Trip Valve (TEV)}

Realizado pela KHI entre 09 e 21/12/12 e refeito integralmente pela CSN entre $16 / 12 / 13$ e $19 / 12 / 13$

\subsubsection{M-6 Function Test of Electronic Governor}

Realizado pela KHI entre 22 e 28/12/13 e refeito pela CSN entre 13/12/13 e 16/12/13

\subsubsection{M-7 Combined Operation Test of Control System}

Realizado pela KHI em 28/12/12 e refeito pela CSN em 19/12/13.

\subsubsection{M-8 Purge}

Realizado pela KHI entre 14/03/13 e 15/03/13 e refeito pela CSN entre 09/12/13e $12 / 12 / 13$

\subsubsection{M-9 Auxiliary Equipment}

Realizado pela KHI nos equipamentos de seu fornecimento entre 09/12/13 e 28/12/12 e entre 08/03/13 e 12/03/13 e não refeito pela CSN. Os equipamentos de fornecimento da CSN (CSN Supply) foram checados pela GGEN.

\subsection{Testes de Instrumentação}

Concluído pela KHI entre 10/12/12 e 28/12/12 e refeitos pela CSN a partir de 23/12/13 até $03 / 01 / 14$

\subsubsection{Testes elétricos}

Concluído pela $\mathrm{KHI}$ entre 10/12/12 e 28/12/12 e refeitos pela CSN a partir de 23/12/13 até $03 / 01 / 14$

\subsection{Comissionamento a Quente}

Mecânicos M-1 a M-4, Elétricos E-1 e E-2 e Mecânicos M-5 a M-10

\subsubsection{M-1 Purge Test}

Realizado pela KHI entre 14/03/13 e 15/03/13 e refeito pela CSN em 11/12/13. 


\subsubsection{M-2 Bypass Valves}

Realizado parcialmente pela $\mathrm{KHI}$ entre $14 / 03 / 13$ e 15/03/13 e refeito pela CSN entre 09/12/13 e 16/12/13.

- Confirm the normal operating of bypass valves, and including controllability of TIP by MBV.

- Confirm that pressure fluctuation of BFG line such as top press, and downstream.

- Adjustment of BSV opening limit by detecting the pressure fluctuation of BFG line by trip operation.

\subsubsection{M-3 Turbine Start-up and M-4 Over-speed}

Realizado pela CSN entre 16/12/13 e 19/12/13.

- Confirm the normal operating of each parts in increasing speed step by step as shown in TRT Test Operating Schedule.

- Confirm the necessary data for automatic sequential start-up.

- Confirm that over speed trip device shall be acted at the speed at $110 \%$ (3.960rpm) an rated (3.600rpm).

- Confirm the automatic sequential start-up and stop up to synchrony stand-by speed (3.620rpm).

\subsubsection{E-1 AVR Adjustment}

Realizado pela CSN entre 02/01/14 e 03/01/14

- AVR adjustments shall be carried out under the rated speed.

\subsubsection{E-2 Parallel Running (at test position of 52G)}

Concluído pela CSN em 09/01/14

- Confirm that generator circuit breaker $(52 \mathrm{G})$ shall be closed at its test position by manual and automatic operating for a few times.

- Confirm that 52G shall be closed at its normal position by manual and automatic operating.

\subsubsection{M-5 Loading \& Load Rejection and M-6 Trip}

Realizado pela CSN entre 13/01/14 e 15/01/14

- Confirm the normal operating of each part in increasing generator output step by step as shown in TRT Test Operating Schedule.

- Conform the following matter in case of load rejection and the emergency stop (trip).

\subsubsection{M-7 Automatic Sequence, M-8 Heat Running e M-9 Noise Measurement}

Realizado pela CSN em 16/01/14

- Finally, confirm the normal operating of all automatic sequential controls including bypass valves, turbine start-up, loading, continuous operating and stop.

- Confirm that TRT can operate for five hours under the maximum continuous load.

- Measure the noise around TRT

\section{REINÍCIO DOS TESTES}

- Localizado em 06/12/13, o software de controle do Governor foi de imediato copiado e com tal cópia, entre 09 e 11/12/13, foram realizados testes de lógica de 
todos os componentes do sistema, além da movimentação física, modo manual, do SB - Stator Blade e da TEV - Turbine Emergency Valve.

- Também em 11/12/13 foi realizada purga do sistema para permitir que em 12/12/13 ocorresse o primeiro giro do conjunto turbina/gerador, conforme definido no documento KHI 92142.0610.04 R1, a 800rpm.

- Em 16/12/13 foi efetuada uma alteração na cópia do software de controle impondo a abertura da MBV antes da BSV e em seguida um teste operacional de ambas, em modo manual e automático.

- Em 17/12/13 foram verificadas as condições de reset da turbina.

- Em 19/12/13 foi realizado um teste de rotação até a nominal, 3.600rpm, seguida de trip da TRT, a pressão de $0,6 \mathrm{~kg} / \mathrm{cm} 2$ e $\mathrm{SB}$ em automático. Também foi realizado teste de trip em sobre-velocidade, a 3.700 rpm, nas mesmas condições de pressão.

- Nos dias subsequentes os testes foram realizados com o SB na condição automática sendo que a pressão de entrada de gás na TRT foi sendo gradualmente aumentada até um delta $P$ de $0,30 \mathrm{~kg} / \mathrm{cm} 2$ em relação à pressão de topo do AF3.

- Entre 23 e 28/12/13 foram realizados os testes elétricos (dielétricos e de malhas de comando, controle e proteção) dos equipamentos do sistema de potência Gerador, TTR, GP (generator panel), cubículos B-8082 (52S - Supplier) e 52G (generator). Neste dia também foram iniciados os testes de auto start e trip da TRT, prejudicados por uma parada não programada do AF3 e concluídos nos dias subsequentes.

- Em 27/12/13, com o fechamento do disjuntor 52S, ligado diretamente à barra $\mathrm{B}$, do CMC 80, dos Moto Sopradores, ocorreu a energização reversa do TTR. O equipamento foi monitorado por $24 \mathrm{~h}$ e nesse período foram retiradas amostras de óleo mineral para testes.

- Em 02/01/14 foi identificada e corrigida a causa da atuação intempestiva da proteção do TTR, ocorrida em 02/01/14. Nesta mesma data foi executada a $1^{\circ}$ Etapa do AVR - Average Voltage Regulation, que significa girar o conjunto turbina/gerador à velocidade nominal, 3.600rpm, excitar o gerador, em modos manual e automático, até ser atingida a tensão de $13,8 \mathrm{kV}$, mantido aberto 0 disjuntor 52G, ou seja, sem sincronizar o gerador com a rede de $13,8 \mathrm{kV}$ da UPV. A pressão de entrada foi de aproximadamente $2,0 \mathrm{~kg} / \mathrm{cm} 2$. Foi implantada na lógica de controle a segurança de sobrepressão no topo e na entrada da TRT p/ TRIP.

- Em 03/01/14 foi realizada a $2^{\circ}$ Etapa do AVR, que significa, além das ações acima, fechar o $52 \mathrm{G}$ na posição de teste, nos modos manual e automático e naquelas condições provocar trips da TRT.

- Em 06/01/14 foi elaborado um Plano de Contingência para a redução possíveis impactos sobre a produção dos AF2 e 3 em caso de atuação de algum disjuntor do CMC 80 ou até mesmo do 88 , do AF2, durante os testes de sincronismo e de carga do gerador.

- Em 07/01/14 foram realizadas manobras de cargas e controle do Moto Soprador 2, da barra B para a Barra A do CMC80, alinhados os turbos sopradores 1 e 2, respectivamente para os AF 2 e 3 e em seguida normalizados os sopros para os AF 2 e 3 a partir dos Moto Sopradores. Às 10h:30min a TRT foi operada, mas não foi possível garantir a sequência de fases no disjuntor $52 \mathrm{G}$ para uma sincronização segura do gerador com o método de medição de tensão nos TP Transformadores de Potencial. 
- Em 08/01/14 foi invertida a sequência de fases dos cabos de potência do gerador e nova tentativa de medição de faseamento foi realizada, mas sem sucesso.

- Em 09/01/14, utilizando o método do círculo do fogo, foi garantida a condição de sincronismo nos modos manual e automático. Etapa E-1.

- Em 13/01/14 a TRT voltou a ser operada e o gerador excitado até a geração de 2,2MW e em seguida 5,5MW. Em ambas as situações foram realizados testes de rejeição de carga, com acompanhamento de seus possíveis efeitos na barra B do CMC80, tanto quanto a possíveis desarmes de disjuntores, quanto por influência na tensão da barra. Resultado altamente satisfatório.

- Em 14/01/14 configurados os valores de vibração restantes (8:00/10:00) pela AssetCare, rodou-se a TRT, feita a sincronização, o ajuste do Fator de Potência c/ 5,5 MW, preenchido os dados da Planilha de Testes e feito Teste de TRIP (Geral).

- Em 15/01/14 rodou-se a TRT, sincronizou-se, elevou-se a geração até $11 \mathrm{MW}$, feito teste de TRIP (2X), depois elevou-se a geração até 16,5 MW (Dados: Vazão de Sopro $=6500 \mathrm{Nm} 3 / \mathrm{h} ; \mathrm{VGAF}=633.000 \mathrm{Nm} 3 / \mathrm{h} ; \mathrm{Ptop}=2,2 \mathrm{~kg} / \mathrm{cm} 2 ; \mathrm{P}$ entrada TRT $=1,9 \mathrm{~kg} / \mathrm{cm} 2 ; \mathrm{SB}=60 \%$ aberto) e feito teste de TRIP (Total) $2 X$.

- Em 16/01/14 rodou-se de novo a TRT e deixou-se operando de modo contínuo (Start up concluído). Elevada a P topo de 2,2 para 2,45 kg/cm2, mantido o $\Delta \mathrm{P}$ da TRT em 0,30, alcançado Max. 18,4 MW (com a MBV fechada) com SB aberto $55 \%$, sendo a $\mathrm{VGAF}=633.042 \mathrm{Nm} 3 / \mathrm{h}(\mathrm{VAS}=6500 \mathrm{Nm} 3 / \mathrm{min})$.

- Em 17/01/14 iniciada a Operação Assistida, com a TRT gerando 16,5 a 17 MW no 1 o turno.

- A Operação Assistida foi finalizada em 24/01/14 quando já se obtinha $18 \mathrm{MW}$ de geração de energia (dentro da META do Plano de alcançar nesta Etapa no mínimo $80 \%$ da capacidade nominal da Planta).

- Quanto a limpeza das palhetas da TRT, a Planta da ETE TRT encontrava-se em operação, porém ainda em fase de ajustes sem, contudo, prejudicar a operação da TRT.

\section{RESULTADOS}

No dia 16/01/2014 a Turbina de Topo entrou em operação de forma contínua e definitiva. No gráfico abaixo podemos ver o perfil de geração no período de janeiro/2014.

No período de 17/01 a 24/01/14, testes adicionais foram realizados no sentido de avaliar e garantir a performance pela TRT. Nas condições operacionais atuais do AF3, com geração média entorno de $18 \mathrm{MWh}$.

Em 14/05/14, excepcionalmente, o AF3 foi operado em condições próximas de seus parâmetros de projeto (pressão de topo $2,5 \mathrm{~kg} \times 650 \mathrm{dam} 3 / \mathrm{h}$ ), resultando em uma geração de $21,25 \mathrm{MW}$.

As situações acima permitiram afirmar que a TRT atingiu o desempenho previsto na curva KHI de geração x pressão x vazão de GAF conforme figura 9. 


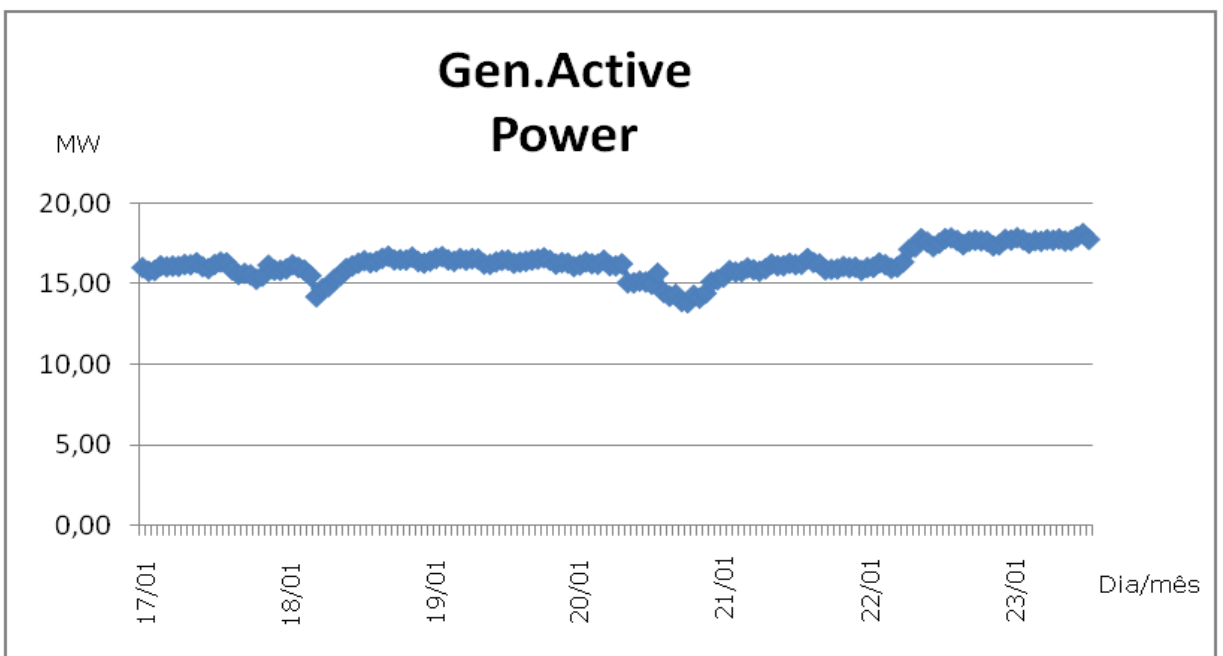

Figura 9. Gráfico de controle de geração de energia (período de 17 a 23-01-2014).

\subsection{Financeiros}

A energia elétrica média gerada pela TRT permite fornecer energia para mais de 73.000 residências, ou aproximadamente $88 \%$ dos domicílios de Volta Redonda. Dados estes retirados do Censo do IBGE de 2010 e consumo médio de Energia Elétrica domiciliar de dez/2013 informado pela ANEEL que está em 182,73KWh/mês no sudeste brasileiro.

A geração da TRT possui uma contribuição significativa no total da UPV que pode ser visto na figura 10 . Esta contribuição chega a $4 \%$ da energia da UPV.

\section{GERACÃO / DISTRIBUICÃO ENERGIA ELÉTRICA UPV - $60 \mathrm{HZ}$}

$$
\text { TOTAL UPV }=240.765 \mathrm{MWh}
$$

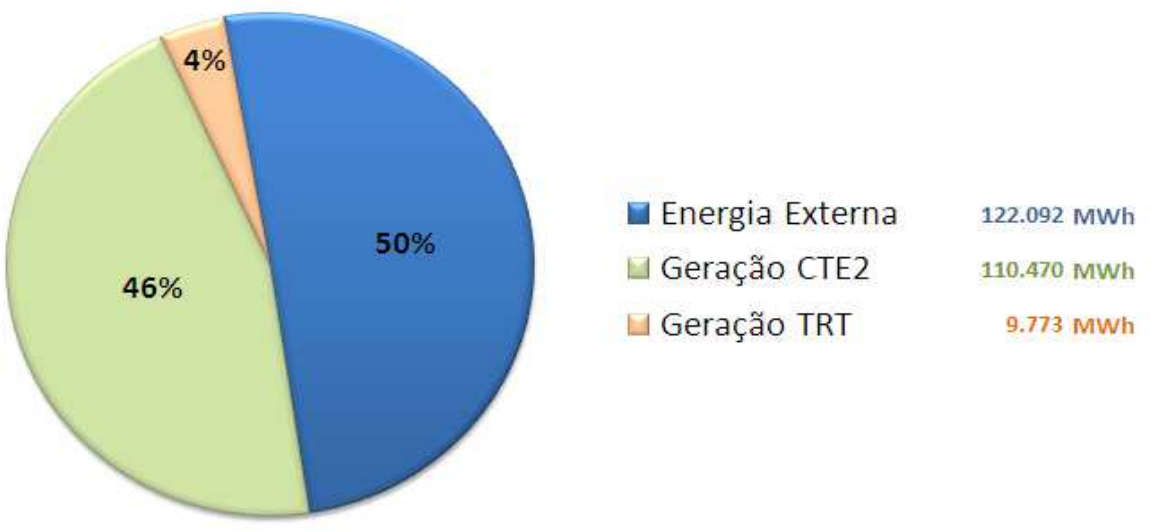

Figura 10. Gráfico de comparação da distribuição da energia elétrica na CSN-UPV

Os valores ao longo do ano desde janeiro de 2014 podem ser vistos na figura 11 abaixo. 


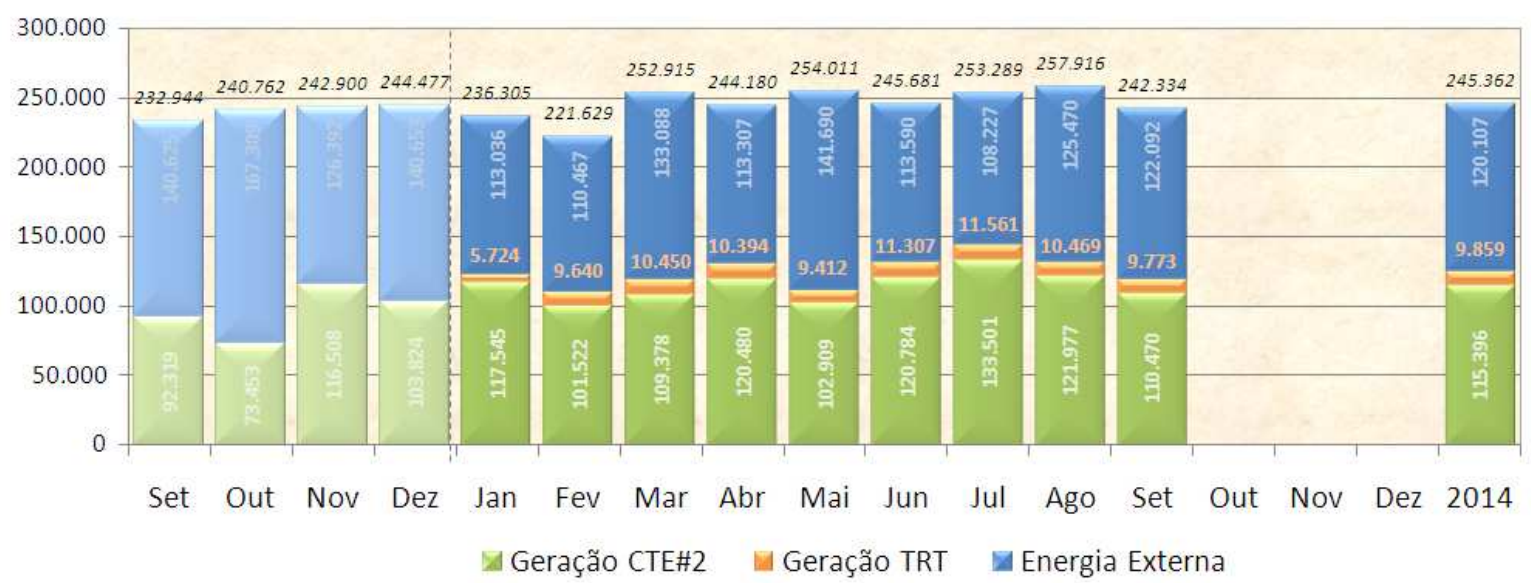

Figura 11. Contribuição de janeiro até setembro da geração da TRT

Os ganhos da geração de energia elétrica da TRT podem ser calculados baseandose nos valores comerciais praticados de mercado e dos contratos de longo prazo estabelecidos com as concessionárias. Abaixo na tabela 1 temos extratificados os cálculos de e seus ganhos.

\begin{tabular}{|c|c|c|c|c|c|c|c|c|c|c|c|c|c|c|c|c|c|c|c|c|}
\hline Meses & & $\mathrm{jan} / 14$ & & fev/14 & & $\operatorname{mar} / 14$ & & $a b r / 14$ & & mai/14 & & jun/14 & & $\mathrm{jul} / 14$ & & ago/14 & & set/14 & & out/14 \\
\hline MWh -Acumulado & & 5.724 & & 9.858 & & 10.740 & & 10.339 & & 9.379 & & 11.490 & & 11.899 & & 10.059 & & 10.469 & & 9.912 \\
\hline $\begin{array}{c}( \\
\text { Operação/Manutençã } \\
\text { o, etc...) }\end{array}$ & RS & $165.512,63$ & RS & $218.789,76$ & RS & $309.421,68$ & RS & $312.613,33$ & RS & $332.818,31$ & RS & $310.086,09$ & RS & $360.271,08$ & RS & $388.195,02$ & RS & $407.705,00$ & RS & $339.327,00$ \\
\hline Custo E.E. Ext. & RS & 88,54 & RS & 97,32 & RS & 102,75 & RS & 103,09 & RS & 79,16 & RS & 92,39 & RS & 87,66 & RS & 89,96 & RS & 92,23 & RS & 93,06 \\
\hline $\begin{array}{c}\text { Preço Energia } \\
\text { Elétrica Externa } \\
\text { (RS/MWh) }\end{array}$ & RS & 378,22 & RS & 822,63 & RS & 822,83 & RS & 822,83 & RS & 815,33 & RS & 417,16 & RS & 592,54 & RS & 707,37 & RS & 728,95 & RS & 776,88 \\
\hline
\end{tabular}

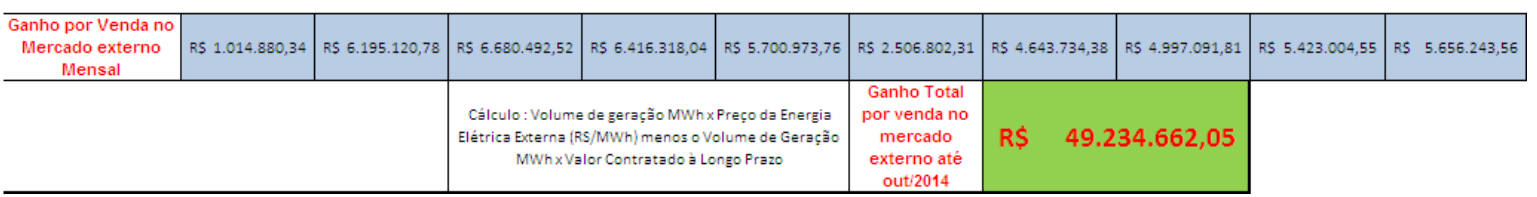

Figura 12. Ganhos Financeiros [Fonte: CSN-GGCE/ASSA].

A comercialização da energia hora excedente pela geração da TRT passa a ser um ativo de comercialização no mercado externo de energia, sendo assim tivemos um ganho real pela venda no mercado externo sendo este valor já próximo a 50 milhões, oportunidade esta, somente propiciada pela antecipação da entrada da TRT em operação.

\subsection{Tecnológicos}

O desenvolvimento do trabalho de partir a TRT sem a presença dos especialistas da Kawasaki ficou extremamente claro os ganhos de know-How da equipe que participou e o empenho de cada equipe. A diretriz de dar prosseguimento sem o fabricante mostra a confiança na capacidade técnica do grupo CSN/Engenharia, vindo de encontro aos requisitos necessários para enfrentar as disparidades e desafios de mercado hoje existentes. 


\section{CONCLUSÃo}

A geração de $20 \mathrm{MWh}$ redunda na disponibilidade desta energia para outras utilizações no Estado do Rio de Janeiro, uma vez que a Fornecedora Light passa a somar esta reserva de energia em seus barramentos.

A energia gerada é totalmente limpa, o que reduz a necessidade de geração desta energia por outras fontes que impactam no meio ambiente, com por exemplo: combustão, hidroelétrica.

A tecnologia adquirida durante o desenvolvimento dos projetos, fabricação, montagem, testes, comissionamentos e posta em marcha, agregou conhecimentos específicos a todos os especialistas da CSN e Empresas Contratadas envolvidos neste empreendimento.

Além dos pontos positivos acima enumerados, a geração de aprox. $20 \mathrm{MWh}$ de energia nos remete a planejar o retorno de todo o investimento neste projeto, em apenas poucos anos de operação contínua.

Podemos concluir que o projeto de implantação de um conjunto Turbina de Recuperação de Pressão do Topo / Gerador, do Alto Forno \#3 da CSN, atingiu as metas preconizadas na abertura dos estudos deste empreendimento e ainda, passou a contribuir com a geração de energia sustentável e sem causar nenhum tipo de impacto ambiental.

\section{Agradecimentos}

Agradecemos à Deus pelas oportunidades de aprendizagem, à todas as pessoas que participaram do trabalho de partida da TRT, sem a sinergia do grupo isso não seria possível.

\section{BIBLIOGRAFIA}

Manual de Operação, Manutenção, Engenharia da Turbina de Topo Kawasaki

Documento $\mathrm{KHI}$ de referência:

92142-0610.01 - Test and Adjusting Manual for Pre-Commissioning (Cold Commissioning)

92142-0620.01 - Test and Adjusting Report for Pre-Commissioning (Cold Commissioning)

Documento KHI de referência:

92142-0610.04 - Test and Adjusting Manual for Commissioning (Hot Commissioning)

92142-0620.04 - Test and Adjusting Report for Commissioning (Hot Commissioning) 